\title{
ON NOWHERE DIFFERENTIABLE FUNCTIONS BEING A CONVOLUTION OF CONTINUOUS FUNCTIONS ${ }^{1}$
}

\author{
WITOLD BOGDANOWICZ
}

In this paper we shall consider the problem of differentiability of convolutions of continuous functions. This problem arose in connection with Mikusiński's method of developing operational calculus and also in connection with differentiability of Fourier series. Mikusiński considers the set $C$ of all functions $x(t)$ continuous for $t \geqq 0$. This is a linear space in which he introduces a multiplication $x * y$ of two elements $x, y$ by means of the convolution

$$
(x * y)(t)=\int_{0}^{t} x(t-s) y(s) d s .
$$

The commutative ring received in that way has, by a theorem of Titchmarsh, the property that $x * y=0$ if and only if either $x=0$ or $y=0$. Therefore, by a well-known theorem of algebra, it can be extended to a field $P$ of formal fractions $w=x / y(y \neq 0)$. If by $l$ we denote the function $l(t)=1$ for $t \geqq 0$, then we see that

$$
(l * x)(t)=\int_{0}^{t} x(s) d s .
$$

If $x$ has continuous derivative then we have

$$
l * x^{\prime}=x-x(0) l .
$$

Denoting by $p$ the element $1 / l$, we have

$$
x^{\prime}=p x-x(0) .
$$

The derivative now is expressed in purely algebraic terms. Hence we may assume the right side of the equation as a definition of a generalized derivative. Now the derivative is defined for all continuous functions and coincides with the usual derivative in the case when it exists and, say, is continuous.

We see that some algebraic operations over continuous functions lead to differentiable functions; for example, the operation $l * x$. Mikusiński asked the question whether the operation $x * x$ leads to a differentiable function (for $t>0$ ). The question was answered by

Received by the editors August 6, 1962 and, in revised form, August 14, 1963.

1 This work was supported by the National Science Foundation under Grant No. P-8174, G-16003. 
Jarnik who has proved that there exists a continuous function $x$ such that the convolution $x * x$ is nowhere differentiable (except at the point $t=0$ ). In this paper we shall prove more general theorems concerning that convolution.

In the theory of Fourier series we consider the convolution of periodical functions

$$
(x * y)(t)=\int_{0}^{2 \pi} x(t-s) y(s) d s .
$$

This convolution has the property that if

$$
x(t)=\sum_{n} a_{n} e^{i n t}, \quad y(t)=\sum_{n} b_{n} e^{i n t}
$$

then

$$
(x * y)(t)=2 \pi \sum_{n} a_{n} b_{n} e^{i n t} .
$$

Hence in this case theorems on nondifferentiability of convolution of continuous functions may be expressed as theorems concerning nondifferentiability of Fourier series of continuous functions.

1. We shall use the method of category to prove our theorems. Such a method of treatment of nondifferentiable functions is due to Banach who has shown using this method that the set of all nowhere differentiable functions is a residual one in the space of all continuous functions.

Let $x$ be a function continuous on an interval $I=[a, b]$. Let us put

$$
f(x)=\inf _{s \in I}\left\{\frac{|x(t)-x(s)|}{|t-s|}: t \in I, t \neq s\right\} .
$$

Proposition 1. The functional $f(x)$ has the following properties:

(a) $0 \leqq f(x) \leqq \infty$.

(b) $f(\lambda x)=|\lambda| f(x)$ for any real $\lambda$.

(c) If $y$ satisfies Lipschitz's condition with a constant $L$ then $f(x+y)$ $\leqq f(x)+L$.

(d) If $x$ is differentiable at a point $s \in I$ then $f(x)<\infty$.

(e) $f(x)=0$ if and only if $x(t)$ is a constant on $I$.

(f) If $X$ is a metric space of continuous functions such that convergence of a sequence of functions $x_{n}$ to a function $x$ in $X$ implies uniform convergence of the sequence on the interval $I$, then the functional $f(x)$ considered on the space $X$ is lower semi-continuous on it.

(g) Let $a=s_{0}<s_{1}<\cdots<s_{m}=b$ and $x$ be a function differentiable on the interval $I$. Let 


$$
M=\inf \left\{\frac{\left|x\left(s_{n}\right)-x\left(s_{n-1}\right)\right|}{\left|s_{n}-s_{n-1}\right|}: n=1,2, \cdots, m\right\}
$$

then

$$
f(x) \geqq M \text {. }
$$

Proof. Properties (a), (b) are obvious. Let $y$ satisfy Lipschitz's condition with the constant $L$.

We have

$$
\frac{|(x(t)+y(t))-(x(s)+y(s))|}{|t-s|} \leqq \frac{|x(t)-x(s)|}{|t-s|}+L,
$$

and from that inequality we get property (c).

If $x$ is differentiable at a point $s \in I$ then the function

$$
g(t)=\frac{x(t)-x(s)}{t-s} \quad \text { for } t \in I, t \neq s
$$

is bounded but this implies that $f(x)$ is finite.

Let us prove now property (e). If $x$ is a constant function then it is obvious that $f(x)=0$. Conversely, if $f(x)=0$ then there exists a sequence $s_{n} \in I$ such that the sequence

$$
\sup \left\{\frac{\left|x(t)-x\left(s_{n}\right)\right|}{\left|t-s_{n}\right|}: t \in I, t \neq s_{n}\right\}=h_{n}
$$

tends to zero when $n$ tends to infinity. From that we have the condition

$$
\left|x(t)-x\left(s_{n}\right)\right| \leqq h_{n}\left|t-s_{n}\right| \quad \text { for } t \in I, t \neq s_{n}, n=1,2, \cdots .
$$

The sequence $s_{n}$ may be supposed to be convergent to a number $s_{0} \in I$. Then we shall have

$$
\left|x(t)-x\left(s_{0}\right)\right| \leqq 0 \quad \text { for } t \in I, t \neq s_{n}, n=1,2, \cdots .
$$

From the continuity of $x(t)$ on the interval $I$ we get also that

$$
x(t)=x\left(s_{0}\right) \quad \text { for all } t \in I .
$$

Let us prove property (f). We have to show that the set of all $x \in X$ such that $f(x) \leqq a$, for a constant $a$, is closed in the space $X$. Let us take any sequence $x_{n}$ from the set and let $x_{n}$ converge to $x$ in the space $X$.

We have

$$
f\left(x_{n}\right) \leqq a
$$


Let $\epsilon_{n}$ be a sequence of positive numbers and let $\epsilon_{n}$ tend to zero. There must exist a sequence $s_{n} \in I$ such that

$$
\sup _{t \in I}\left\{\frac{\left|x_{n}(t)-x_{n}\left(s_{n}\right)\right|}{\left|t-s_{n}\right|}: t \in I, t \neq s_{n}\right\} \leqq a+\epsilon_{n},
$$

hence

$$
\left|x_{n}(t)-x_{n}\left(s_{n}\right)\right| \leqq\left(a+\epsilon_{n}\right)\left|t-s_{n}\right| \text { for } t \in I, t \neq s_{n}, n=1,2, \cdots \text {. }
$$

The sequence $s_{n}$ may be supposed to be convergent to a point $s_{0} \in I$. Since $x_{n}(t)$ tends to $x(t)$ uniformly on the interval $I$, we have

$$
\left|x(t)-x\left(s_{0}\right)\right| \leqq a\left|t-s_{0}\right| \quad \text { for } t \in I, t \neq s_{n}, n=1,2, \cdots .
$$

From the continuity of $x$ on the interval $I$ we see that the last inequality holds for all $t \in I$, which implies that $f(x) \leqq a$.

To prove the last property let us prove the following lemma. Let $x(t)$ be differentiable on an interval $\left[s_{0}, s_{1}\right]$. Let $x\left(s_{0}\right)=x\left(s_{1}\right)=0$. Then, for any $s_{0} \leqq s \leqq s_{1}$, either there exists $s_{0} \leqq t \leqq s_{1}$ different from $s$ and such that $x(t)=x(s)$, or the derivative $x^{\prime}(s)=0$.

Proof. The function $x(t)$ being continuous on the interval $\left[s_{0}, s_{1}\right]$ assumes its maximum at a point $t_{1}$ and its minimum at a point $t_{2}$. Let us take any $s$ from the interval $\left[s_{0}, s_{1}\right]$. We may assume that $s \neq s_{0}$, $s \neq s_{1}$ and $0 \neq x(s) \neq x\left(t_{1}\right)$ and $x(s) \neq x\left(t_{2}\right)$ as in any of the cases the condition of the lemma is satisfied. Therefore we may have either $x(s)>0$ or $x(s)<0$. Let us consider the first case. The second one can be considered in the same way. We have

$$
0=x\left(s_{1}\right)=x\left(s_{0}\right)<x(s)<x\left(t_{1}\right) .
$$

We see that $s$ must belong either to the interval $\left(s_{0}, t_{1}\right)$ or to the interval $\left(t_{1}, s_{1}\right)$. If $s$ belongs to $\left(s_{0}, t_{1}\right)$ then we can find a point $t$ in the interval $\left(t_{1}, s_{1}\right)$ such that $x(t)=x(s)$. If $s$ belongs to $\left(t_{1}, s_{1}\right)$ then we shall find a point $t$ in the interval $\left(s_{0}, t_{1}\right)$ such that $x(t)=x(s)$. The lemma is proved. To prove that $f(x)$ has the last property it is enough to show that

$$
\sup _{t}\left\{\frac{|x(t)-x(s)|}{|t-s|}: t \in I, t \neq s\right\} \geqq \frac{\left|x\left(s_{i}\right)-x\left(s_{i-1}\right)\right|}{\left|s_{i}-s_{i-1}\right|}
$$

for all $s$ from the interval $\left[s_{i-1}, s_{i}\right]$. Consider the function

$$
y(t)=x(t)-\frac{x\left(s_{i}\right)-x\left(s_{i-1}\right)}{s_{i}-s_{i-1}}\left(t-s_{i-1}\right)-x\left(s_{i-1}\right)
$$

for $t$ in the interval $\left[s_{i-1}, s_{i}\right]$. This function satisfies the conditions of 
our lemma. Hence, for any $s$ from the interval $\left[s_{i-1}, s_{i}\right]$, there exists another point $t$ such that $y(t)=y(s)$ or $y^{\prime}(s)=0$. In the first case we have that

$$
\frac{x(t)-x(s)}{t-s}=\frac{x\left(s_{i}\right)-x\left(s_{i-1}\right)}{s_{i}-s_{i-1}},
$$

and in the second one we have

$$
x^{\prime}(s)=\frac{x\left(s_{i}\right)-x\left(s_{i-1}\right)}{s_{i}-s_{i-1}} .
$$

But this proves that the inequality (i) must be satisfied.

2. Let $C_{1}$ denote the space of all periodical continuous functions $x$ with the period $\tau=2 \pi$. $C_{1}$ with the norm

$$
\|x\|=\sup \{|\dot{x}(t)|: 0 \leqq t \leqq 2 \pi\}
$$

is a Banach space. In this space we define a convolution by formula (B).

THEOREM 1. The set $E$ of all functions $x \in C_{1}$ such that the functions

$$
x, x * x, x * x * x, \cdots
$$

are nowhere differentiable is residual in the space $C_{1}$.

Proof. Let $F$ denote the complement of the set $E$ in the space $C_{1}$. Let

$$
F_{m n}=\left\{x \in C_{1}: \underset{(n \text { timea) }}{f(x)} \underset{*}{* \ldots}\right\}
$$

We see that

$$
F \subset \bigcup_{m, n=1}^{\infty} F_{m n} .
$$

Since $f$ is a lower semi-continuous functional on the space $C_{1}$ and the operation $y=x * \cdots * x$ as an operation from $C_{1}$ into itself is continuous (it follows from the inequality $\|x * y\| \leqq 2 \pi\|x\|\|y\|$ ), we get that the sets $F_{m n}$ are closed. Hence to prove the theorem it is sufficient to show that no set $F_{m n}$ has interior points.

LEMMA. If $F_{m n}$ has interior points then there exists a constant $M$ such that

$$
f(\underset{\text { (n times) }}{* \ldots x}) \leqq M\|x\| n \quad \text { for all } x \in C_{1} .
$$


Proof. Suppose that $F_{m n}$ has interior points, that is, the set $F_{m n}$ contains a sphere

$$
K(y, r)=\left\{x \in C_{1}:\|x-y\|<r\right\} .
$$

Since the set of differentiable functions is dense in the space $C_{1}$, we may assume that $y$ has continuous derivative and hence satisfies Lipschitz's condition with a constant $L$. We have

$$
f(x * \cdots * x) \leqq m \quad \text { for } x \in K(y, r) \text {. }
$$

We see, that for $x \in K(y, r)$, we have $\|x\| \leqq\|y\|+r=d$, and that $y * x * x * \cdots * x$ satisfies Lipschitz's condition with the constant $L(2 \pi d)^{n-1}$. Let $\bar{x}=x-y$. From property (c) of the functional $f$ we get

$$
f(\bar{x} * x * x * \cdots * x) \leqq f(x * x * \cdots * x)+L(2 \pi d)^{n-1}
$$

for all $\bar{x} \in K(0, r)$ and $x=y+\bar{x}$, and so

$$
f(\bar{x} * x * \cdots * x) \leqq m+L(2 \pi d)^{n-1} .
$$

We may prove in that way by induction that there exists a number $d$ such that

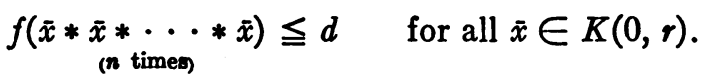

We have $\bar{x}=(r / 2\|x\|) x \in K(0, r)$ for $x \neq 0$, and from the linearity of the convolution $x * y$ with respect to each variable $x, y$ and from the homogeneity of the functional $f$, we see that

$$
f\left(\left(\frac{r}{2\|x\|}\right)^{n} x * x * \cdots * x\right) \leqq d,
$$

and finally that for $M=(2 / r)^{n} d$

$$
f(x * x * \cdots * x) \leqq M\|x\|^{n} .
$$

This inequality should hold for all $x \in C_{1}$ (it obviously holds when $x=0$ ).

Now we see that in order to prove that the set $F_{m n}$ has no interior points it is sufficient to show that the condition of the lemma is not satisfied. Let $x$ be $\cos (k t)$. We have $\cos (k t)=\frac{1}{2} e^{i k t}+\frac{1}{2} e^{-i k t}$. Hence $x * x=\pi x$, and

$$
x * \cdots * x=\pi^{n-1} x \text {. }
$$

(n times)

From property $(\mathrm{g})$ of the functional $f$ we see that if we take $s_{i}=i \pi / k$ $(i=0,1, \cdots, 2 k)$ we get that 


$$
\frac{2 k}{\pi} \leqq f(x) .
$$

Therefore, if the condition of the lemma was satisfied, we would have that

$$
2 \pi^{n-2} k \leqq f(x * x * \cdots * x) \leqq M\|x\| n=M \quad \text { for } k=1,2,3, \cdots
$$

But this is impossible. Thus the set $F$ is contained in a set which is the union of a countable collection of closed sets none of which has interior points. This means that the set $E$ is residual in the space $C_{1}$.

CoRollary 1. For every $\bar{x} \in C_{1}$ and $r>0$ there exists a continuous function $x \in C_{1}$ such that

$$
|x(t)-\bar{x}(t)|<r \quad \text { for all. } t
$$

and all functions

$$
x, x * x, x * x * x, \cdots
$$

are nowhere differentiable.

This corollary follows from the fact that a residual set is dense in the space, so there has to exist a point $x \in E$ such that $\|x-\bar{x}\|<r$.

Similarly one can prove the following theorem.

TheOREM 2. For every sequence of continuous functions $\bar{x}_{n} \in C_{1}$ and every sequence $r_{n}$ of positive numbers, there exists a sequence of continuous functions $x_{n} \in C_{1}$ such that $\left\|x_{n}-\bar{x}_{n}\right\|<r_{n}$ for $n=1,2, \cdots$, and all the functions of the form

$$
x_{n_{1}} * x_{n_{2}} * \cdots * x_{n_{k}},
$$

where $n_{1}, \cdots, n_{k}$ are positive integers, are nowhere differentiable.

The proof of the theorem we leave to the reader.

If functions $x$ and $y$ satisfy Hölder's condition with powers $\alpha$ and $\beta$ respectively and $\alpha+\beta>1$, then the convolution $x * y$ is everywhere differentiable. We shall prove a theorem which shows that it cannot happen in general if the sum of the powers is less than 1.

Let $H^{\alpha}$ denote the set of all functions $x \in C_{1}$ such that they satisfy Hölder's condition with all powers $\beta>0$ less than $\alpha$. $H^{\alpha}$ may be considered as an $F$-space if we introduce in it semi-norms by the formula

$$
\|x\|_{n}=\sup \left\{\frac{|x(t)-x(s)|}{|t-s|^{\beta_{n}}}: t \neq s\right\}+|x(0)|,
$$

where $\beta_{n}$ is an increasing sequence of positive numbers tending to $\alpha$. 
Proposition 2. The set of all functions satisfying Lipschitz's condition is dense in the space $H^{\alpha}(0<\alpha \leqq 1)$.

Proof. The sets of the form

$$
V_{n e x}=\left\{y:\|y-x\|_{n}<\epsilon\right\}
$$

form a base of neighborhoods of the point $x$ in the space $H^{\alpha}$. Therefore to prove the proposition it is enough to show that every such set contains a function which satisfies Lipschitz's condition.

Let $\beta_{n+1}-\beta_{n}=\gamma$ and let $\delta$ be so small that

$$
4\|x\|_{n} \delta^{r}<\epsilon .
$$

Let $m$ be a positive integer such that $2 \pi / m<\delta$ and let

$$
t_{j}=\frac{j}{m} 2 \pi \quad \text { for } j=0, \pm 1, \pm 2, \cdots .
$$

Let $y(t)$ be continuous and linear on each interval $\left[t_{j}, t_{j+1}\right]$, and let

$$
y\left(t_{j}\right)=x\left(t_{j}\right) \quad \text { for } j=0, \pm 1, \pm 2, \cdots .
$$

The function $y$ satisfies Lipschitz's condition and for any $s$ and $t$ such that $s-t \geqq \delta$ we have

$$
\begin{aligned}
& |(y(t)-x(t))-(y(s)-x(s))| \\
& \leqq\left|y(t)-y\left(t_{j}\right)\right|+\left|x(t)-x\left(t_{j}\right)\right|+\left|y(s)-y\left(t_{k}\right)\right|+\left|x(s)-x\left(t_{k}\right)\right|,
\end{aligned}
$$

where $t_{j}$ is the nearest point of the sequence (i) to the point $t$ such that $t<t_{j}$, and $t_{k}$ is the nearest point of the sequence (i) to the point $s$ and such that $t_{k}<s$. We see that

$$
\left|x(t)-x\left(t_{j}\right)\right| \leqq\|x\|_{n+1}\left|t-t_{j}\right|^{\beta_{n}+\gamma} \leqq \frac{\epsilon}{4}|t-s|^{\beta_{n}}
$$

and

$$
\left|x(s)-x\left(t_{k}\right)\right| \leqq \frac{\epsilon}{4}|t-s| \beta_{n} .
$$

We have also

$$
\begin{aligned}
\left|y(t)-y\left(t_{j}\right)\right| & =\frac{\left|x\left(t_{j-1}\right)-x\left(t_{j}\right)\right|}{\left|t_{j-1}-t_{j}\right|}\left|t-t_{j}\right| \leqq\|x\|_{n+1} \frac{\left|t_{j-1}-t_{j}\right| \beta_{n}+\gamma}{\left|t_{j-1}-t_{j}\right|} \\
& \leqq\|x\|_{n+1}\left|s-t_{2}\right|_{n}+\gamma \leqq \frac{\epsilon}{4}|s-t| \beta_{n} .
\end{aligned}
$$

In the same way we shall see that 


$$
\left|y(s)-y\left(l_{k}\right)\right| \leqq \frac{\epsilon}{4}|s-t|^{\beta_{n}} .
$$

Thus

$$
|(y(s)-x(s))-(y(t)-x(t))| \leqq \epsilon|s-t|^{\beta_{n}}
$$

if $s-t \geqq \delta$, but when $s-t<\delta$ the inequality is obvious, so we have finally

$$
\|y-x\|_{n}<\epsilon
$$

and this is what we were to prove.

Theorem 3. Let $0<\alpha_{i}$ and $\alpha_{1}+\cdots+\alpha_{k} \leqq 1$. The set $E$ of all $y=\left(x_{1}, \cdots, x_{k}\right)$ in the space $H^{\alpha_{1}} \times \cdots \times H^{\alpha_{k}}$ such that the function

$$
x_{1} * x_{2} * x_{3} * \cdots * x_{k}
$$

is nowhere differentiable is residual in the space $H^{\alpha_{1}} \times \cdots \times H^{\alpha_{m}}$.

Proof. Let $F$ be the complement of the set $E$. We have

$$
F \subset \bigcup_{m},
$$

where

$$
F_{m}=\left\{y=\left(x_{1}, \cdots, x_{k}\right): f\left(x_{1} * \cdots * x_{k}\right) \leqq m\right\} .
$$

As the convergence in the space $H$ is stronger than uniform convergence, we see that the sets $F_{m}$ are closed in the space $H^{\alpha_{1}} \times \cdots$ $X H^{\alpha_{k}}$. Using the proposition we can prove that if $F_{m}$ had interior points then there would exist a constant $M$ and an index $n$ such that

$$
f\left(x_{1} * \cdots * x_{k}\right) \leqq M\|y\|_{n}^{k} \quad \text { for all } y,
$$

where $\|y\|_{n}=\sup \left\{\left\|x_{1}\right\|_{n}, \cdots,\left\|x_{k}\right\|_{n}\right\}, y=\left(x_{1}, \cdots, x_{k}\right)$.

This condition is not satisfied. As before it is enough to consider the functions

$$
x_{j}(t)=\cos p t, \quad p=1,2, \cdots ; j=1, \cdots, k .
$$

In the same way we may prove a stronger theorem.

Theorem 4. Let $0<\alpha_{i}$ and $\alpha_{1}+\cdots+\alpha_{k} \leqq 1$. Then the set $E$ of all $y=\left(x_{1}, \cdots, x_{k}\right)$ in the space $H^{\alpha_{1}} \times \cdots \times H^{\alpha_{k}}$ such that all the functions

$$
x_{p_{1}} * \cdots * x_{p j},
$$

where $p_{1}<p_{2}<\cdots<p_{j}<k$, are nowhere differentiable is residual in the space $H^{\alpha_{1}} \times H^{\alpha_{2}} \times \cdots \times H^{\alpha_{1}}$. 
3. Let $C$ denote the space of all functions $x(t)$ continuous for $t \geqq 0$. In this space we define semi-norms by

$$
\|x\|_{n}=\sup \{|x(t)|: 0 \leqq t \leqq n, n=1,2, \cdots\} .
$$

The space $C$ with the family of semi-norms is an (F)-space. We introduce the convolution by the formula

$$
(x * y)(t)=\int_{0}^{t} x(t-s) y(s) d s .
$$

Theorem 5. The set $E$ of all functions $x \in C$ such that the functions

$$
x, x * x, x * x * x, \cdots
$$

are nowhere differentiable in the interval $(0, \infty)$ is residual in the space $C$.

Proof. Let $F$ be the complement of the set $E$. Let

$$
F_{m n k}=\left\{x \in C: f_{k}(x * \ldots * x) \leqq m\right\},
$$

where $f_{k}$ is defined for the interval $I_{k}=\left[k^{-1}, k\right]$. We see that

$$
F \subset U F_{m n k} \quad(m, n, k=1,2,3, \cdots) .
$$

Since the convergence in the space $C$ is uniform on each closed interval and the operation $x * x * \cdots * x$, as an operation from $C$ into itself is continuous, we see that the sets $F_{m n k}$ are closed. The sets

$$
V_{n \epsilon}(y)=\left\{x \in C:\|x-y\|_{n}<\epsilon\right\}
$$

form a base of neighborhoods of the point $y$. Hence, if a set $F_{m n k}$ had interior points, then it would contain a set $V_{q \epsilon}(y)$. We might suppose $y$ satisfied Lipschitz's condition on every finite interval. As in Theorem 1 we should see that there would exist a number $M$ such that

$$
f_{k}(x * \ldots * x) \leqq M\|x\|_{q}^{n} \quad \text { for all } x \in C,
$$

and hence

$$
f_{k}(x * \ldots * x)
$$

would be bounded for all $x$ of the form

$$
x_{p}(t)=\sin p k t, \quad p=1,2, \cdots .
$$

But this cannot happen according to the following lemma.

Lemma. Let 


$$
y_{p}=x_{p} * \cdots * x_{p}
$$

Then

$$
\lim _{p} f_{k}\left(y_{p}\right)=\infty .
$$

Proof. We have (see Operational calculus by Mikusinski)

$$
y_{p}(t)=\frac{1}{2^{2 n-1}} \frac{1}{a^{2 n-2}}\left[A_{2 n}(a t) a^{-1} \sin a t-B_{2 n}(a t) t \cos a t\right]
$$

where $a=p k \pi$ and the polynomials $A_{n}(S), B_{n}(S)$ are defined by the formulas

$$
\begin{aligned}
A_{1}(S) & =1, \quad A_{2}(S)=1, \quad B_{1}(S)=0, \quad B_{2}(S)=1, \\
A_{n+1}(S) & =\frac{2 n-1}{n} A_{n}(S)-\frac{S^{2}}{n(n-1)} A_{n-1}(S), \quad n=2,3,4, \cdots, \\
B_{n+1}(S) & =\frac{2 n-1}{n} B_{n}(S)-\frac{S^{2}}{n(n-1)} B_{n-1}(S), \quad n=2,3,4, \cdots .
\end{aligned}
$$

From the formulas one may prove that the polynomial $B_{2 n}(S)$ has the order $2 n-2$. Let us divide the interval $\left[k^{-1}, k\right]$ by means of the points

We have

$$
t_{m}=\frac{m}{k p}, \quad p \leqq m \leqq k^{2} p .
$$

$$
\begin{aligned}
& \left|\frac{y_{p}\left(t_{m}\right)-y_{p}\left(t_{m-1}\right)}{t_{m}-t_{m-1}}\right| \\
& \quad=\frac{1}{2^{2 n-1}} \frac{1}{(k \pi)^{2 n-2}} \frac{1}{p^{2 n-2}}\left|B_{2 n}(m \pi) m+B_{2 n}((m-1) \pi)(m-1)\right| .
\end{aligned}
$$

Since $B_{2 n}(u)$ is a polynomial of the order $2 n-2$, we have for suffciently large $p$ the estimation

$$
\left|B_{2 n}(m \pi) m+B_{2 n}((m-1) \pi)(m-1)\right| \geqq A p^{2 n-1} \quad \text { for } m \geqq p,
$$

where $A$ is a positive number. Therefore

$$
\left|\frac{y_{p}\left(t_{m}\right)-y_{p}\left(t_{m-1}\right)}{t_{m}-t_{m-1}}\right| \geqq C p \quad \text { for all } m \geqq p,
$$

where $C$ is a positive number. By Proposition 1 property (g) this implies that $f_{k}\left(y_{p}\right)$ tends to infinity when $p$ tends to infinity and the lemma is proved. 


\section{REFERENCES}

1. H. Auerbach and S. Banach, Über die Höldersche Bedingung, Studia Math. 3 (1931), 180-184.

2. S. Banach, Über die Baire'sche Kategorie gewisser Funktionenmengen, Studia Math. 3 (1931), 174-179.

3. W. Bogdanowicz, Sur la methode de Banach appliquee d la demonstration de l'existence d'une fonction continue n'ayant nulle part de dérive et Etant une convolution de fonctions continue, Colloq. Math. 7 (1959), 120-121.

4. V. Jarnik, Sur le produit de composition de deux fonctions continues, Studia Math. 12 (1951), 58-64.

5. S. Kaczmarz, Integrale vom Dini'schen Typus, Studia Math. 3 (1931), 189-199.

6. S. Mazurkiewicz, Sur l'integrale $\int_{0}^{1} t^{-1}[f(x+t)+f(x-t)-2 f(x)] d t$, Studia Math. 3 (1931), 114-118.

7. J. Mikusiśski, Operational calculus, Pergamon Press, New York, 1959.

8. S. Ruziewicz, Ein Beispiel sur Hölderschen Bendingung, Studia Math. 3 (1931), 185-188.

9. H. Steinhaus, Anwendungen der Funktionanalysis auf einige Fragen der reellen Funktionentheorie, Studia Math. 1 (1929), 51-81.

10. K. Zeller, FK-Räume in der Funktionentheorie. I, II, Math. Z. 58 (1953), 288-305, 414-435.

11. A. Zygmund, Trigonometric series, Cambridge Univ. Press, Cambridge, 1959.

UNIVERSITY OF MARYLAND AND

University of Warsaw, Warsaw, Poland 